\title{
Bessel Companding Transform for reducing PAPR, OBI and BER in OFDM Systems
}

\author{
B.Naga siva Prasad ${ }^{1}$ Jyothi $^{2}$, Dr.V.Sailaja ${ }^{3}$ \\ ${ }^{1}$ (ECE, Godavari Institute of Engineering \& Technology/Jntuk, India) \\ ${ }^{2}$ (ECE, Godavari Institute of Engineering \& Technology/Jntuk, India) \\ ${ }^{3}$ (ECE, Godavari Institute of Engineering \& Technology/Jntuk, India)
}

\begin{abstract}
Orthogonal Frequency division multiplexing (OFDM) is a very popular technique in wireless communications which provides excellent robustness to channel fading and immune to interference. OFDM is one of a popular modulation technique for transmitting large amounts of digital data over radio carriers. One of the major problems that had been observed in this is the Peak-To Average power Ratio (PAPR). PAPR is a distortion that had occurred due to large number of sub carriers that are used in OFDM for modulating the digital data. Many techniques were available to reduce the PAPR in OFDM systems, among them the companding technique can be used to enhance the OFDM transmission performance by decreasing the PAPR effectively and hence BER. Here we proposed "Bessel Companding Algorithm" to reduce this significant drawback in OFDM Systems.
\end{abstract}

Keywords : Orthogonal Frequency Division Multiplexing (OFDM), peak to average power ratio (PAPR), bit error rate (BER), Out Band Interference (OBI), Additive White Gaussian Noise (AWGN), Bessel Companding Algorithm, Quadrature Amplitude Modulation (QAM) and Multi Carrier Modulation (MCM).

\section{Introduction:}

OFDM is a key broad band wireless technology which supports data rates in excess of (100 Mbps) with high spectral efficiency, immunity to multipath fading and simple in implementation using fast Fourier transform (FFT). It is basically a multicarrier modulation (MCM) technique which depends on Orthogonality principle. Orthogonality means it allows the sub carriers which are orthogonal to each other as shown in fig (1). OFDM system converts a frequency selective fading channel into a set of parallel narrow band flat-fading channels across the sub carriers, which is readily implemented by present day processors in many high speed networks. The rapidly growing application of OFDM includes WiMAX, DVB/DAB, 4G and IEEE $802.11 \mathrm{a} / \mathrm{g} / \mathrm{n}$ wireless systems.

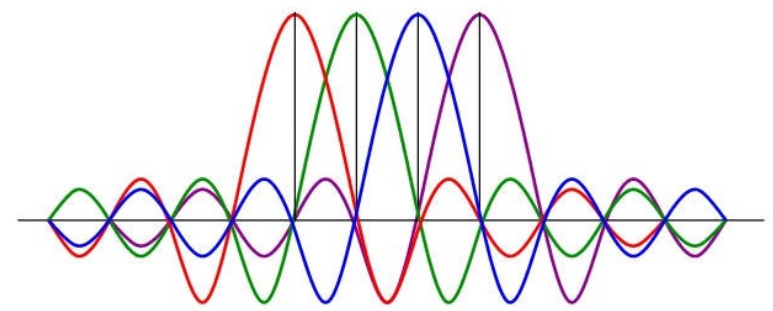

Fig (1)

It has some drawbacks compared to single carrier systems. One of the major drawbacks is high peak-to-average power ratio (PAPR). In these systems the PAPR increases with increase in number of sub carriers (N). Hence PAPR in an OFDM system can be significantly higher. High PAPR in an OFDM system essentially arises because of the IFFT operation at the transmitter as shown in fig (2), i.e. data symbols across sub carriers will add and produce a high peak value signal. When the peak deviation above average is significantly high the signal level moves outside the dynamic linear range of the analog/digital circuits (amplifiers, A/D and D/A converters), hence high PAPR in OFDM results in amplifier saturation leading to inter carrier interference (ICI). To tackle this we are using the Bessel companding technique to reduce the PAPR effectively. 


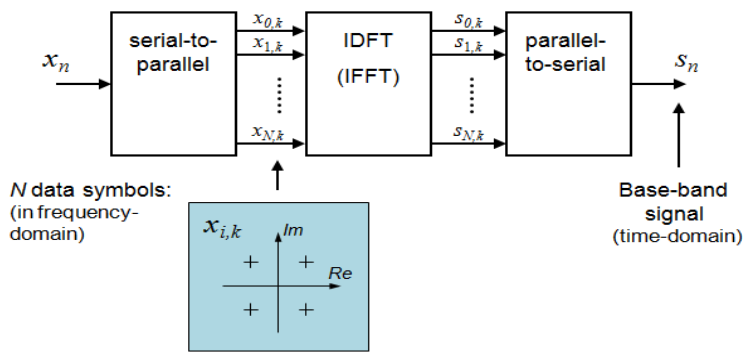

Fig (2)

\section{Schematic Of Ofdm System:}

OFDM divides the available wide band among a set of orthogonal overlapping sub carriers i.e. the relationship between all the carriers must be carefully controlled to maintain the orthogonality principle. A single stream of data is split into several parallel bit streams each of which is coded and modulated on to a subcarrier (i.e. IFFT operation).

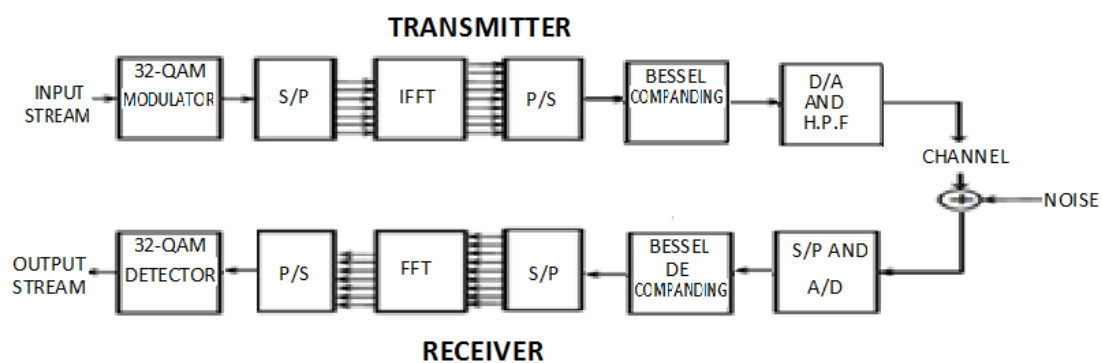

Fig (3)

This scheme of generating the composite transmit OFDM signal employing IFFT/IFT was proposed by two scientists namely Weinstein and Ebert in 1971, both were scientists at Bell Telephone Laboratories. The proposed scheme of generating the MCM transmit signal at much lower implementation complexity when compared with bank of modulators in conventional MCM systems. After the IFFT operation, the data is converted from parallel to serial using a Shift register, which is companded and transmitted through the channel. On the receiver side the information is recovered by employing decompanding and FFT/DFT, which is converted back into serial. Now the serial data is demodulated and is used for further processing as shown in fig (3).

\section{Peak-To-Average Power Ratio In Conventional Ofdm Systems:}

High Peak-to-Average Power Ratio is one of the major practical problems involved in OFDM modulation. High PAPR results from the modulation itself i.e. the IFFT operation where multiple subcarriers are added together to form the signal to be transmitted. When ' $N$ ' sinusoids are added, the peak magnitude would have a value of 'N'. High PAPR signals are usually undesirable as it strains the analog circuitry. High PAPR signals would require a large range of dynamic linearity for the analog circuits which usually results in expensive devices and high power consumption with lower efficiency (for e.g. power amplifier has to operate with larger back-off to maintain linearity).

Let $X(0), X(1), X(2) \cdots, X(N-1)$ represent the data sequence to be transmitted in an OFDM symbol with subcarriers. The baseband representation of the OFDM symbol is given as

$$
X(t)=\frac{1}{\sqrt{N}} \sum_{n=0}^{N-1} X_{n} e^{\frac{j 2 \pi n t}{N}} \quad ; 0 \leq t \leq T
$$

Where, ' $\mathrm{T}$ ' is the duration of the OFDM symbol.

When the peak deviation above average is significantly high the signal level moves outside the dynamic linear range of the analog/digital circuits (amplifiers, A/D and D/A converters). Hence high PAPR in OFDM results in amplifier saturation.The PAPR of the transmit signal $\mathrm{x}(\mathrm{t})$ is the ratio of the maximum instantaneous power to the average power. 
Continuous time PAPR:-

$$
\text { PAPR }=\frac{M A X\left[|x(t)|^{2}\right]}{E\left[|x(t)|^{2}\right]}
$$

The large amplitude variation increases in-band noise and hence increases in Bit Error Rate (BER). The PAPR in OFDM systems is characterized by using the complementary cumulative distribution function (CCDF). CCDF curves gives information regarding the strength of PAPR in OFDM signals to be transmitted. It is used to measure the effectiveness of PAPR reduction, which is the probability that PAPR exceeds some threshold $\left(p_{0}\right)$.

\section{$\mathrm{CCDF}=$ Probability $\left(\mathrm{PAPR}>\boldsymbol{p}_{0}\right)$}

\section{Companding:}

Companding is also one of the signal distortion techniques which decrease PAPR and minimized OBI while reducing bit error rate (BER) efficiently. So it can be used to improve OFDM system performance. Signal is first converted into time domain by using IFFT/IDFT, then companded, after that it is converted to analog before transmitting it through channel. Due to companding, the quantization error for large signals is significantly large which degrades the BER performance of the system. So the companding technique improves the PAPR in expense of BER performance of the system.

Here we are comparing the proposed Bessel companding technique with one of the popular exponential companding.

\section{(a) Exponential companding :}

A new nonlinear companding technique, namely "exponential companding" that can effectively reduce the PAPR of transmitted (companded) OFDM signals by transforming the statistics of the amplitudes of these signals into uniform distribution. The new scheme also has the advantage of maintaining a constant average power level in the nonlinear companding operation.

Let $|t(n)|^{d}$, the $d^{\text {th }}$ power of the amplitude of companded signal $t(n)$, have a uniform distribution in the interval $[0, \alpha]$. The exponent ' $d$ ' is called the degree of a specific exponential companding scheme.

Where the companding function

$$
h(x)=\operatorname{sign}(x(n)) \cdot \sqrt[d]{\alpha\left[1-\exp \left(-\frac{|x(n)|^{2}}{\sigma^{2}}\right\}\right]}
$$

Where $\operatorname{sign}(\mathrm{x}(\mathrm{n}))$ is the sign function. The positive constant ' $\alpha$ ' determines the average power of output signals. In order to keep the input and output signals at the same average power level.

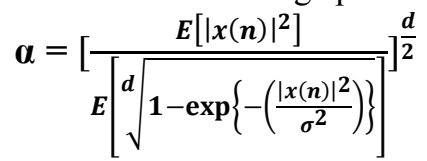

At the receiver side, we use the inverse function of $h(x)$ for decompanding, given by

(b) Bessel companding:

$$
h^{-1}(\mathrm{x})=\operatorname{sign}(\mathrm{x}(\mathrm{n})) \sqrt{-\sigma^{2} \log _{e}\left[1-\frac{x(n)^{d}}{\alpha}\right]}
$$

Here we proposed a companding algorithm which is the second order Bessel differential equation

$$
\frac{d^{2} y}{d x^{2}}+x \frac{d y}{d x}+\left(x^{2}-n^{2}\right) y=0
$$

The solution for the equation gives the Bessel function:

$$
\begin{gathered}
y_{n}(x)=k_{1} j_{n}(x)+k_{2} y_{n}(x) . \\
\text { Where, } j_{n}(x)=\frac{1}{\pi} \int_{0}^{\pi} \cos (n t-x \sin (t)) d t, \\
y_{n}(x)=\frac{j_{n}(x) \cos (n \pi)-j_{-n}(x)}{\operatorname{sinn} \pi} \\
j_{-n}(x)=(-1)^{n} j_{n}(x) .
\end{gathered}
$$

Here, we are normalizing $k_{1}=k_{2}=1$.

Therefore the companding function is given by

$$
f(x)=\beta \cdot \operatorname{sign}(x(n)) .[\operatorname{besselj}(n, 0)-\operatorname{besselj}(n, \alpha .|x(n)|)]
$$

Where $\operatorname{Besselj}(\cdot)$ is the Bessel function of the $n^{\text {th }}$ order, $\alpha$ is the parameter that controls the degree of companding (and ultimately PAPR). $\beta$ is the factor adjusting the average output power of the compander to the same level as the average input power . 
Where

$$
\beta=\sqrt{\frac{E\left[|x(n)|^{2}\right\rceil}{E\left[\mid \operatorname{besselj}(n, 0)-\operatorname{besselj}\left(n, \alpha .|x(n)|^{2}\right)\right]}}
$$

Where $\mathrm{E}[\cdot]$ denotes the expectation.

The decompanding function is the inverse of $\mathrm{f}(\mathrm{x})$ used at the receiver section, given by

$$
\mathbf{f}^{-1}(x)=\frac{1}{\propto} \cdot \operatorname{sign}(x(n)) \cdot \operatorname{besselj}^{-1}\left[n, \operatorname{besselj}(n, 0)-\frac{|x(n)|}{\beta}\right]
$$

Notice that the input to the decompander is a quantized signal with finite set of values as shown in fig (4).

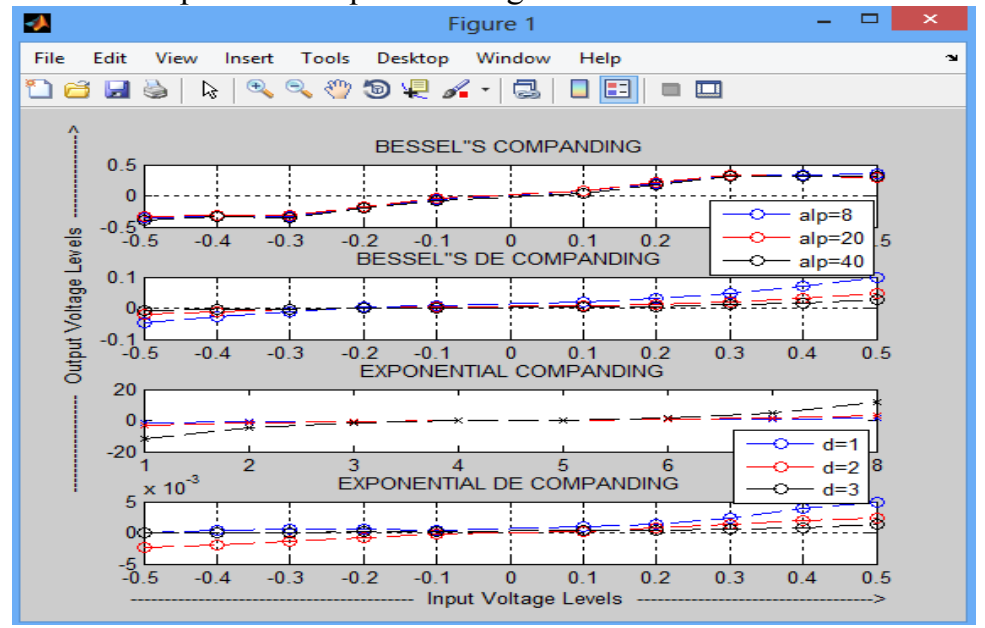

$\operatorname{Fig}(4)$

\section{PAPR Simulation:}

The major drawback in OFDM system is high PAPR (Peak To Average Power Ratio). High PAPR signals required a large range of dynamic linearity of the analog circuits which usually results in expensive devices and high power consumption with lower efficiency. Hence high PAPR in OFDM results in amplifier saturation. In OFDM system, some input sequences results in higher PAPR than others. For example, an input sequences that requires all such carriers to transmit their maximum amplitudes would certainly result in a high output PAPR. Thus by limiting the possible input sequences to a smallest sub set, it should be possible to obtain output signals with a low PAPR. We define PAPR of the transmit signal $x(t)$ as, it is the ratio of the maximum instantaneous power to the average power.

PAPR in OFDM systems are characterized using the CCDF ('Complementary Cumulative Distribution Function'). CCDF vs. PAPR for the companding schemes are shown in fig (5).

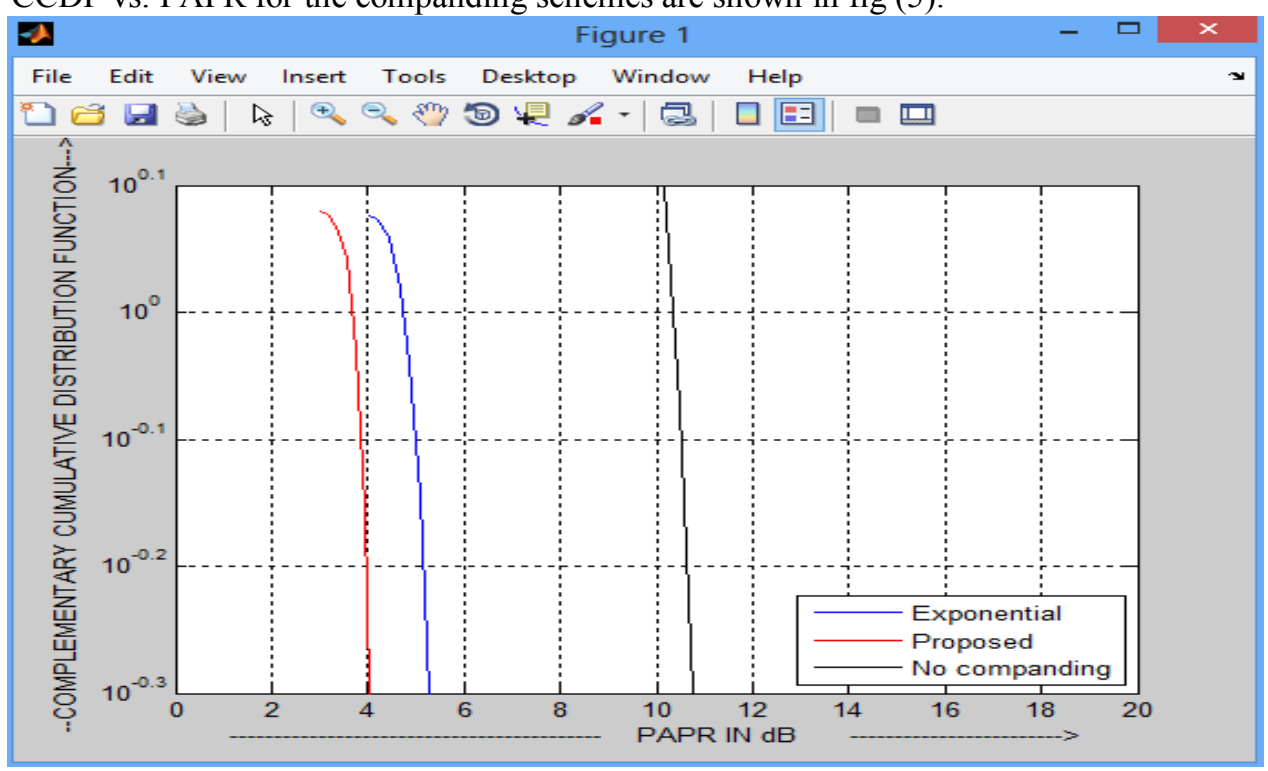

$\operatorname{Fig}(5)$ 


\section{BER Simulation:}

Let $y(n)$ denote the output signal of the compander, where $w(n)$ are the samples of AWGN signal $w(t)$. The received signal can be expressed as $\mathrm{f}(\mathrm{x}(\mathrm{n}))=\mathrm{y}(\mathrm{n})=\mathrm{r}(\mathrm{n})=\mathrm{t}(\mathrm{n})+\mathrm{w}(\mathrm{n})$. After decompanding operation, we get the resulting equation as follows.

$$
\overline{\boldsymbol{r}}(\mathrm{n})=\boldsymbol{f}^{-1}(\mathrm{r}(\mathrm{n}))=\mathrm{t}(\mathrm{n})+\boldsymbol{f}^{-1}(\mathrm{w}(\mathrm{n}))
$$

By using the first order Taylor series expansion, we can be approximate this $\bar{r}(\mathrm{n})$ as follows

$$
\bar{r}(\mathrm{n})=\mathrm{t}(\mathrm{n})+\left.\frac{d f^{-1}(u)}{d u}\right|_{u=y(n)} \cdot w(n)
$$

From the given Equation shows that if $y(n)$ falls into the range of the decompanding function $\mathrm{f}^{-1}(u)$ where $\left.\frac{d f^{-1}(u)}{d u}\right|_{u=y(n)}<1$, the noise $\mathrm{w}(\mathrm{n})$ is suppressed, and if $\mathrm{y}(\mathrm{n})$ is out of the range, $\left.\frac{d f^{-1}(u)}{d u}\right|_{u=y(n)}>1$ and the noise is enhanced. Therefore, if the parameter ' $\alpha$ ', ' $n$ ' (order of Bessel function ) is properly chosen such that more $y(n)$ is within the noise-suppression range of $f^{-1}(u)$, it is possible to achieve better overall BER performance, where transmitted signal $\mathrm{t}(\mathrm{n})$ and received signal $\bar{r}(\mathrm{n})$ are compared for calculating bit error rate. The simulated results for bit error rate vs. signal to noise ratio is as shown in fig (6).

Notice that the signal-to-noise ratio (SNR) in a typical Additive White Gaussian Noise (AWGN) Channel is much greater than 1 . Therefore BER and PAPR affect each other adversely and there must be the tradeoff between these two.

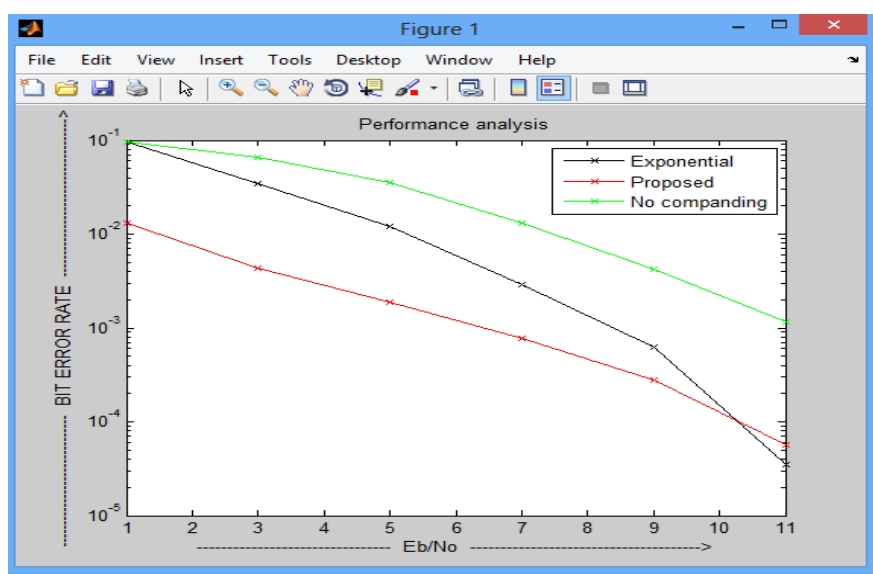

Fig (6)

\section{OBI Simulation:}

Out band interference is the leakage of signal (spectral) energy into the adjacent channels. The knowledge of the power spectral density (PSD) of the companded signal gives the easy analyzation of the OBI caused by companding technique. Unfortunately PSD is in general mathematically intractable for analytical expression, because of the nonlinear companding transform involved. To estimate OBI here we take an alternative approach. Let us consider $\mathrm{f}(\mathrm{x})$ be a nonlinear companding function, and $\mathrm{x}(\mathrm{t})=\sin (\omega \mathrm{t})$ be the input to the compander. The companded signal

$y(t)=f[x(t)]=f[\sin (\omega t)]$ Since $y(t)$ is a periodic function with the same period as $x(t), y(t)$ can then be expanded into the following Fourier series:

Where the coefficients $\mathrm{c}(\mathrm{k})$ is calculated as:

$$
\boldsymbol{y}(\boldsymbol{t})=\sum_{k=-\infty}^{+\infty} \mathbf{c}(\mathbf{k}) \mathrm{e}^{\mathrm{j} k \omega t}
$$

$$
c(k)=c(-k)=\frac{1}{T} \int_{0}^{T} y(t) \mathrm{e}^{-\mathrm{jk} \omega \mathrm{t}} \mathrm{dt} \quad ; \mathrm{T}=\frac{2 \pi}{\omega}
$$

Notice that the input ' $\mathrm{x}$ ' in this case is a pure sinusoidal signal, any c $(\mathrm{k}) \neq 0$ for $|\mathrm{k}|>1$ is the OBI produced by the nonlinear companding process. Therefore, to minimize the OBI, c (k) must approach to zero fast enough as ' $\mathrm{k}$ ' increases. It has been shown that $\mathrm{c}(\mathrm{k}) \cdot \mathrm{k}^{-(\mathrm{m}+1)}$ tends to zero if $\mathrm{y}(\mathrm{t})$ and its derivative up to the $\mathrm{m}$-th order, or in other words, $\mathrm{c}(\mathrm{k})$ converges at the rate of $\mathrm{k}^{-(\mathrm{m}+1)}$. Given an arbitrary number $\mathrm{n}$, the $\mathrm{n}$-th order derivative of $\mathrm{y}(\mathrm{t}), \frac{d^{n} y}{d t^{n}}$, is a function of $d^{i} f(x) / d x^{i},(\mathrm{i}=1,2, \cdots, \mathrm{n})$, as well as $\sin (\omega \mathrm{t})$ and $\cos (\omega \mathrm{t})$, i.e.

$$
c(k)=\frac{d^{n}}{d t^{n}}=g\left(\frac{d^{n} f(x)}{d x^{n}}, \frac{d^{n-1} f(x)}{d x^{n-1}}, \ldots \ldots, \frac{d f(x)}{d x}, \sin (\omega t), \cos (\omega t)\right)
$$


Where $\sin (\omega \mathrm{t})$ and and $\cos (\omega \mathrm{t})$ are continuous functions, $\frac{d^{n} y}{d t^{n}}$ is continous if and only if the condition satisfied $\frac{d^{i} f}{d x^{i}},(\mathrm{i}=1,2, \cdots, \mathrm{n})$ are continuous . Based on this observation we can conclude, Companding introduces minimum amount of OBI if the companding function $f(x)$ is infinitely differentiable.

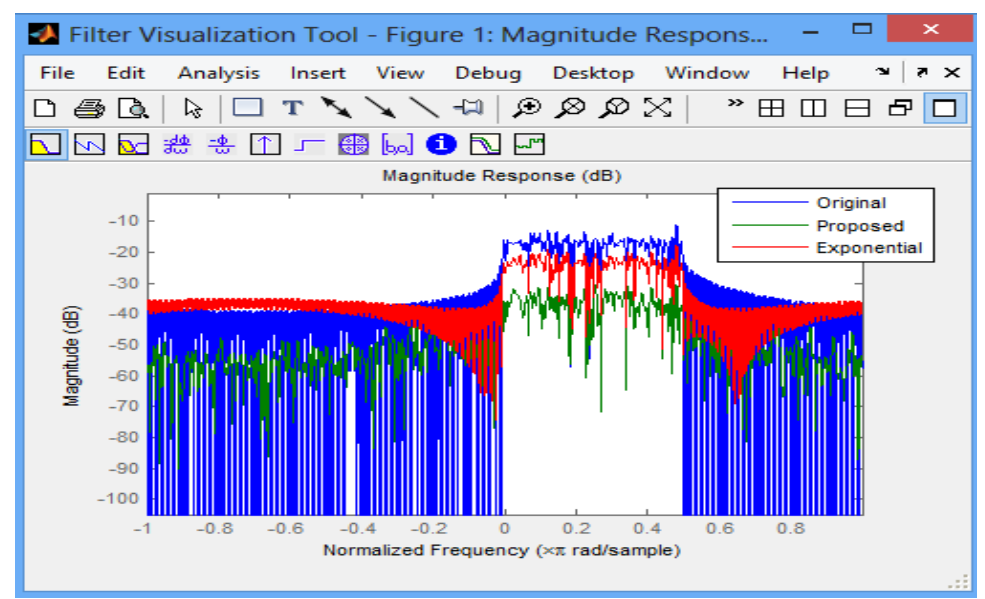

$\operatorname{Fig}(7)$

\section{Simulation Analysis:}

The OFDM system used in the simulation consists of 32 QAM - modulated data points. The size of the FFT/IFFT is 256 . Given the compander input power of $3 \mathrm{dBm}$, the parameter $\alpha$ in the companding function is chosen to be 25 . Consequently about 20 percent of $y(t)$ is within the noise-suppression range of the decompanding function.

One of the popular companding algorithms, namely an exponential companding, is also included in the simulation for the purpose of performance comparison analysis.

CCDF vs. PAPR of the two companding schemes are shown in fig (5). The Bessel companding algorithm nearly gives $(4.0 \mathrm{~dB})$ of PAPR where exponential gives $(5.40 \mathrm{~dB})$. So the proposed companding algorithm improved the PAPR of OFDM system performance.

The BER vs. SNR is plotted in Fig (6). The Bessel companding algorithm gives a BER of (0.000266) at a SNR of $(7 \mathrm{~dB})$, where exponential companding gives a BER of $(0.00281)$ at a SNR of $(7 \mathrm{~dB})$. So the proposed companding algorithm improved OFDM system performance.

The simulated PSD (Power Spectral Density) of the companded signals is illustrated in Fig (7). The Bessel companding algorithm produces $\mathrm{OBI}$ almost $(-33 \mathrm{~dB})$, which is lower than the exponential algorithm, which gives an OBI of $(-19 \mathrm{~dB})$.

One more observation from the simulation is unlike the exponential companding whose performance is found almost unchanged under different degrees of companding; the Bessel companding algorithm is flexible in adjusting its specifications simply by changing the value of ' $n$ ' (the order of the Bessel function) and ' $\alpha$ ' in the companding functions.

\section{Conclusion:}

So, the proposed Bessel companding algorithm improved the performance of OFDM system in terms of PAPR and OBI while reducing BER effectively. Both theoretical analysis and simulation show that the algorithm is very simple, less complex and easy to implement.

\section{References}

[1] B.S. Krongold and D.L.Jones, "PAR Reduction in OFDM via active constellation extension" IEEE Trans. Broadcast. vol. 49, pp. 258-268, Sep. 2003

[2] D. Lowe and X. Huang, "Optimal adaptive hyperbolic companding for OFDM," in Proc. IEEE Second Intl Conf. Wireless Broadband and Ultra Wideband Commun., pp. 24-29, Aug. 2004.

[3] Hikmet Sari and Fabien Buda, "An Analysis of OFDM Peak Power Reduction Techniques for WiMAX Systems" Sequans Communications, 19 Le Parvis, 92073 Paris La Defense, France2009.

[4] I.N.Bronshtein, K. A. Semendyayev, G. Musiol, and H. Muehlig, Handbook of Mathematics, 5th ed. New York: Springer, 2007, p. 422.

[5] J.Akhtman, B.Z. Bobrovsky, and L.Hanzo, "Peak-to-average power ratio reduction for OFDM modems," in Proc. 57th IEEE SemiAnnual Vehicular Technology Conf. (VTC '03), vol. 2, Apr. 2003. pp 1188-1192.

[6] Nishar Ahamed Gugudu, "Evaluation of Channel Coding in OFDM Systems", a thesis in 'Telematics and Signal Processing may 2006. 
[7] R.Anil Kumar, K.Jyothi, Dr.V.Sailaja, "Improved BER and minimized OBI while reducing PAPR by using New Companding Transform", International journal of scientific \& Engineering Research, volume . 3, Issue. 7, july-2012.

[8] R. van Nee and R. Prasad, OFDM for Wireless Multimedia Communications. Boston, MA: Artech House, 2000.

[9] S. H. Han and J. H. Lee, "An Overview of peak-to-average power ratio reduction techniques for multicarrier transmission," IEEE Wireless Commun., vol. 12, pp. 56-65, Apr. 2005.

[10] T. Jiang and Y. Wu, "An overview: peak-to-average power ratio reduction techniques for OFDM signals," IEEE Trans. Broadcast., vol. 54, no. 2, pp. 257-268, June 2008 .

[11] X. Wang, T. T. Tjhung, and C. S. Ng, "Reduction of peak-to-average power ratio of OFDM system using a companding technique," IEEE Trans. Broadcast., vol. 45, no. 3, pp. 303-307, Sept. 1999. 